\title{
Mining Privacy Goals from Privacy Policies Using Hybridized Task Recomposition
}

\author{
JASPREET BHATIA, TRAVIS D. BREAUX, and FLORIAN SCHAUB, \\ Carnegie Mellon University
}

\begin{abstract}
Privacy policies describe high-level goals for corporate data practices; regulators require industries to make available conspicuous, accurate privacy policies to their customers. Consequently, software requirements must conform to those privacy policies. To help stakeholders extract privacy goals from policies, we introduce a semiautomated framework that combines crowdworker annotations, natural language typed dependency parses, and a reusable lexicon to improve goal-extraction coverage, precision, and recall. The framework evaluation consists of a five-policy corpus governing web and mobile information systems, yielding an average precision of 0.73 and recall of 0.83 . The results show that no single framework element alone is sufficient to extract goals; however, the overall framework compensates for elemental limitations. Human annotators are highly adaptive at discovering annotations in new texts, but those annotations can be inconsistent and incomplete; dependency parsers lack sophisticated, tacit knowledge, but they can perform exhaustive text search for prospective requirements indicators; and while the lexicon may never completely saturate, the lexicon terms can be reliably used to improve recall. Lexical reuse reduces false negatives by $41 \%$, increasing the average recall to 0.85 . Last, crowd workers were able to identify and remove false positives by around $80 \%$, which improves average precision to 0.93 .
\end{abstract}

Categories and Subject Descriptors: D.2.1 [Software Engineering]: Requirements/Specifications

General Terms: Design, Languages

Additional Key Words and Phrases: Requirements extraction, crowdsourcing, natural language processing, privacy

\section{ACM Reference Format:}

Jaspreet Bhatia, Travis D. Breaux, and Florian Schaub. 2016. Mining privacy goals from privacy policies using hybridized task recomposition. ACM Trans. Softw. Eng. Methodol. 25, 3, Article 22 (May 2016 ), 24 pages.

DOI: http://dx.doi.org/10.1145/2907942

\section{INTRODUCTION}

Software engineers who design data-intensive systems must increasingly consider the impact of privacy on their software design. Regardless of whether the software is developed using a plan-driven or agile method, software that operates on personal information is often required to be accompanied by a privacy policy, which is a legal document and software artifact that describes consumer data practices. Privacy policies answer important questions about the software's operation, including what personal identifiable information is collected, for what purpose is it used, and with whom is it shared.

This work is supported by the National Science Foundation, under grant no. 1330596.

Authors' addresses: J. Bhatia, T. D. Breaux, F. Schaub, 5000 Forbes Avenue, Institute for Software Research, School of Computer Science, Carnegie Mellon University, Pittsburgh, Pennsylvania, 15213; emails: \{jbhatia, breaux, fschaub\}@cs.cmu.edu.

Permission to make digital or hard copies of part or all of this work for personal or classroom use is granted without fee provided that copies are not made or distributed for profit or commercial advantage and that copies show this notice on the first page or initial screen of a display along with the full citation. Copyrights for components of this work owned by others than ACM must be honored. Abstracting with credit is permitted. To copy otherwise, to republish, to post on servers, to redistribute to lists, or to use any component of this work in other works requires prior specific permission and/or a fee. Permissions may be requested from Publications Dept., ACM, Inc., 2 Penn Plaza, Suite 701, New York, NY 10121-0701 USA, fax +1 (212) 869-0481, or permissions@acm.org.

(c) 2016 ACM 1049-331X/2016/05-ART22 $\$ 15.00$

DOI: http://dx.doi.org/10.1145/2907942 
These policies also serve a US and EU regulatory role to increase data transparency [Reidenberg et al. 2015], and they are used to conduct market surveillance ${ }^{1}$ on industry data practices. The transparency benefits of privacy policies include that consumers can learn how companies use their personal data, while software developers can learn how their third-party service providers protect or put at risk their users' data. User data may be put at risk, for example, when a third-party advertising library acquires that data for use under a more permissive third-party privacy policy.

Methods exist that can be used to help regulators and developers analyze privacy policies. These techniques are based on privacy goals, which describe actions of a system or stakeholder and that are performed on an information type, such as "collecting your e-mail address" or "sharing your location." Goals are also linked to software requirements through goal refinement [Dardenne et al. 1993]. Antón and Earp introduced a privacy-requirements taxonomy based on the manual extraction of privacy goals from privacy policies [Antón and Earp 2004]. Their taxonomy enumerates privacy vulnerabilities that cover threats due to overcollection, storage, and sharing, among others, which engineers can use to perform privacy risk analyses early in the software design life cycle. The taxonomy was implemented in the Privacy Goal Management Tool (PGMT) to later discover how ChoicePoint, a data broker, introduced vulnerabilities by aggregating consumer information across multiple services [Otto et al. 2007]. Breaux et al. [2008] formalized privacy goals in Description Logic, which were adopted by the Eddy privacy specification language for detecting conflicting privacy goals [Breaux et al. 2014] and for tracing dataflows across policies in a service composition [Breaux et al. 2015]. Privacy-goal tracing across policies is especially important when developers want to know how their third parties may use their users' personal information. Finally, privacy goals appear in other requirements-analysis techniques, such as the sociotechnical system-requirements framework for managing confidentiality goals [Paja et al. 2015] and the Nòmos 3 framework [Ingolfo et al. 2014].

These techniques show promise in improving privacy-policy analysis, yet they all depend on the ability to extract privacy goals from policies, which requires significant time and effort. Based on the number of websites browsed by an average user and the time required to read a privacy policy, McDonald and Cranor [2008] estimate that the average user must spend $181 \mathrm{~h}$ to $304 \mathrm{~h}$ per year reading privacy policies. While developers may need to read fewer privacy policies to assess third-party software, the number of services that they rely on can still range from tens to hundreds, depending on the complexity of the service composition [Lin et al. 2012].

To address the challenge of extracting privacy goals from privacy policies, we describe an empirically evaluated framework that facilitates the extraction of privacy goals from privacy policies using a hybrid combination of crowdsourcing and natural language processing (NLP). In our framework, crowd workers provide phrase-level policy interpretations through small 30 s to 60 s tasks, called microtasks. To keep the tasks small and affordable, we employ typed dependency parsing based on part-ofspeech (POS) tagging to compose worker annotations into privacy goals, thus partially automate privacy-goal extraction.

The remainder of this article is organized as follows: in Section 2, we review related work; in Section 3, we introduce our hybrid framework; in Section 4, we present our results from validating the framework with a corpus of five privacy policies; in Section 5 , we discuss the threats to validity; and in Section 6, we present our discussion and future work.

\footnotetext{
${ }^{1}$ Since 2013, the Global Privacy Enforcement Network (GPEN), a global coalition of data-protection agencies, conducts annual privacy-policy sweeps, in which they analyze 1 to 2,000 privacy policies of online services and mobile apps in a concerted effort.
} 


\section{RELATED WORK}

We review related work on requirements extraction from text and from crowdsourcing annotations.

\subsection{Requirements Extraction}

Privacy goals are a semiformal, canonical representation of what data action is performed on which kind of information. The translation from text to formal and semiformal specifications has long been a challenge. Abbott et al. [1983] were among the first to propose mining program descriptions from text for object-oriented design. Later, Antón [1997] introduced the goal-based requirements analysis method (GBRAM) and heuristics to extract goal specifications from text. Goals range from high- and low-level actions to be maintained, achieved, and avoided by the system [Dardenne et al. 1993]. Antón and Earp [2004] applied GBRAM to mine privacy goals from privacy policies, and Breaux and Rao [2013] showed how to extract dataflow requirements from privacy goals described in privacy policies. In this prior work, however, the task of extracting goals from policies required training of expert analysts, as well as high motivation and vigilance that limits the ability to analyze systems of increasing size and complexity. In this article, we extend this prior work with a narrow focus on privacy goals describing the collection, use, transfer, and retention of consumer information with the intent of automating extraction to address the limitations posed by training and expert requirements in prior work.

The majority of the requirements documents and other sources of requirements are written in natural language. Consequently, numerous efforts have been made to utilize NLP techniques to aid the automated analysis of requirements and to perform other $\mathrm{RE}$ tasks [Kof 2004]. These include automatically extracting requirements and goals from text documents, building models from requirements specifications, and identifying ambiguity in requirement documents, among others. Liu et al. [2014] use dependency parsing and predefined pattern matching rules to extract relations needed to build i* strategic dependency models. We also employ dependency parsing in our approach; however, we seek to maximize precision and recall without curating a rule set. Such rule sets greatly improve precision and recall, but at the cost of continuously needing to update the rule set with each new corpora encountered. Casagrande et al. [2014] applied phrase structure grammar to a smart-metering research paper corpus to extract research goals. Their evaluation of the automated method against 44 manually annotated papers produced a precision $=0.1$, and recall $=0.7$. In our work, we achieve similar results using dependency parsing - a related NLP technique-with precision near 0.2 and recall near 0.7 (see Table V). However, our results go further to show that the addition of crowd worker microdata improves precision to 0.64 to 0.79 and recall to 0.69 to 0.96 (see Table VII).

A significant challenge in extracting requirements from text is the problem of ambiguity [Kamsties 2006]. Berry et al. [2003] introduced the Ambiguity Handbook, which describes ambiguity in requirements specifications and legal contracts; they present several strategies for avoiding and detecting ambiguities. Furthermore, object-oriented analysis models of the specified system can be used to identify ambiguities and inconsistencies [Popescu et al. 2008]. More recent work has focused on using machinelearning algorithms based on heuristics drawn from human judgments, to identify nocuous coordination and anaphoric ambiguities in requirements [Yang et al. 2010, 2011]. This approach still requires human interpretation to detect and resolve ambiguity. In our approach, semantic ambiguity arises when certain verbs indirectly indicate company data practices (e.g., "chatting with friends online" can mean that companies "collect chat logs," the latter being less ambiguous about whether collection occurs). To 
discover these practices, we rely on crowd workers to disambiguate the text to identify relevant actions and information types. Because crowd workers vary in their ability to perform these inferences, we rely on crowd-worker consensus to select likely candidates that we then compare to the expert analysis.

\subsection{Crowdsourcing Annotations and Extraction}

Crowdsourcing facilitates tackling problems that remain hard to solve with automated methods by leveraging human intelligence, typically provided by nonexperts [Quinn and Bederson 2011]. Sabou et al. [2012] note that crowdsourcing plays a major role in NLP as an affordable, large-scale means to acquire corpora and train and evaluate extraction methods. Crowdsourced annotations from nonexperts have also been shown to be comparable to expert annotations for certain annotation tasks, such as word similarity, word sense disambiguation, and textual entailment recognition [Snow et al. 2008]. Crowdsourcing has also been employed for requirements elicitation: StakeRare uses social networks and collaborative filtering to elicit and prioritize user requirements [Lim and Finkelstein 2012]. Breaux and Schaub [2014] studied three tasks to extract privacy goals from policies relying only on untrained crowd workers. They show that task decomposition, which requires splitting the goal-extraction task into microtasks, yields better results with lower cost compared to expert analysts. The framework presented herein is based on this observation and employs NLP-based techniques to recompose the microtask results into privacy goals. We now discuss crowdsourcing in extraction-related tasks and challenges in crowdsourcing text annotations.

In order to leverage the potential of crowdsourcing for annotating and extracting information from natural language text, a number of challenges need to be addressed. André et al. [2014] identify major challenges in having nonexperts (novices) perform the annotations, having a transient workforce, and the need to resolve conflicting (and potentially erroneous) annotations. We now discuss these challenges in detail.

Novices have been shown to operate on different levels of abstraction compared to experts [Tanaka and Taylor 1991] and may ignore features that are apparent to experts [Rosch et al. 1976]. In the context of the categorization and representation of physics problems, Chi et al. [1981] find that novices have different mental models than experts and may categorize problems using surface features rather than deep structure. While prior knowledge guides expert reasoning, novices' reasoning may be decontextualized [Shafto and Coley 2003]. This means that it may be more difficult to obtain novice interpretations that match an expert's understanding in areas that require prior knowledge, yet it also provides the opportunity to gain insights on how laypersons reason about certain topics [André et al. 2014]. This theoretical basis informs our decision to choose a decomposition approach, in which the goal mining task is decontextualized into microtasks that do not require expert knowledge about privacy goals and vulnerabilities.

Task design plays an important role in obtaining high-quality annotations from crowd workers. Tasks are usually decomposed into smaller microtasks for crowdsourcing [Allahbakhsh et al. 2013]. Microtasks can be assigned to multiple workers and individual annotations can be aggregated with different approaches [Hung et al. 2013] to obtain annotation reliability based on consensus. Reducing ambiguity and complexity of individual tasks and their instructions further improves result quality [Quinn and Bederson 2011]. Cheating can be discouraged by ensuring that cheating would require as much effort as completing the task honestly [Allahbakhsh et al. 2013]. Willett et al. [2012] further suggest providing meaningful examples and featureoriented prompts, using detailed questions to focus the crowd workers' attention, and using highlighting and preannotations where appropriate. 
A difficult problem is the aggregation of multiple worker annotations, as well as the recombination of answers obtained from multiple microtasks into a coherent interpretation. Statistics can be used to remove outliers, but workers can also help in validating aggregated results [Sabou et al. 2012]. Hung et al. [2013] compared multiple result aggregation strategies and distinguished between noniterative approaches, in which aggregation occurs after all annotations have been collected, and iterative approaches, in which the analysis of annotations influences the issuance of additional tasks, that is, new tasks are issued to address weaknesses in previously collected task data. They find that expectation maximization and supervised learning from multiple experts achieve the highest accuracy and are robust against cheaters. Zhang et al. [2013] propose a positive label frequency threshold approach, which is similar to majority voting but takes into account that labels may be noisy and imbalanced due to certain response biases. Verroios and Bernstein [2014] propose Context Trees to recursively combine local summaries into a global interpretation of complex data. CrowdForge is a general-purpose framework for crowdsourcing complex and dependent tasks with a map-reduce approach, in which workers can decide how to subdivide a task [Kittur et al. 2011]. Result aggregation can be automated or partially performed by workers.

\section{TASK RECOMPOSITION FRAMEWORK}

We now introduce our task recomposition framework, which combines human text interpretation with automated NLP to recompose crowd worker microtask data into partial goal specifications based on the Eddy specification language [Breaux et al. 2014]. We present an example specification in Eddy's SQL-like syntax: the P indicates a permission, which is followed by a canonical description of the action COLLECT, the information type email-contacts, the source keyword FROM and the actor from whom the information is collected (zynga-users), and, finally, the purpose keyword FOR followed by the purpose:

\section{P COLLECT email-contacts FROM zynga-users FOR anything.}

These specifications are formalized in Description Logic, which is used to detect conflicts in policies [Breaux and Rao 2013]. In this article, we aim to recompose microtask data to create a partial goal specification that includes the action and information type. We leave the actor and purpose for future work. We chose Eddy as our target language for expressing privacy goals, because it provides techniques for formally tracing dataflows across privacy policies [Breaux et al. 2015]. However, manual extraction of the prerequisite privacy goals from policies to enable this traceability across thousands of policies is cost-prohibitive. The approach described herein aims to address this limitation.

Figure 1 provides an overview of our hybrid framework that consists of two kinds of manual tasks (square boxes): tasks performed by an analyst once (white boxes), or tasks performed by the crowd workers (red boxes); and automated steps performed by tools (circles) and a reusable lexicon (parallelogram). The arrows point in the direction of dataflows, for example, illustrating where crowd worker annotations are sent to automated tasks; the solid versus dotted lines signify separate but overlapping flows. We now discuss each step in more detail.

During steps 1 and 2, the analyst prepares the input text to the NLP tools used in steps Y, B1, and B2 and the crowd worker platform, in this case Amazon Mechanical Turk (AMT), which is used in steps 3 and 4 . These steps are performed manually by an analyst, once for each policy at present, because they require relatively little time (a few minutes per policy). For step 1, the input text begins as a text file, which may be extracted from an HTML or PDF document. For step 2, the analyst itemizes the text into paragraphs, averaging 90 to 120 words, that can be annotated in less than $1 \mathrm{~min}$ 


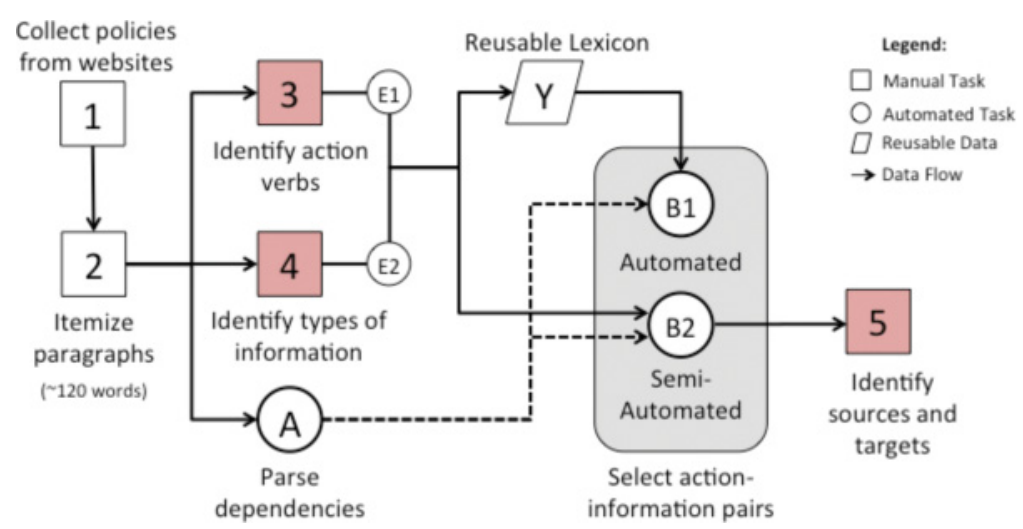

Fig. 1. Task recomposition workflow; red boxes represent crowd worker tasks.

by crowd workers, while ensuring that each paragraph's context remains undivided. For example, the analyst ensures that lists are not separated across tasks, and that anaphoric references, such as "it" or "this," are contained in the same paragraph as the noun phrases to which they refer. This invariant can lead to paragraphs that exceed 120 words, which is balanced by smaller 50- to 60 -word paragraphs. The 120 word average limit determines the average time required by one worker to annotate a paragraph, which we set to $60 \mathrm{~s}$. This average time provides workers small, but frequent, microbreaks between tasks and allows workers frequent opportunities to stop annotating text whenever they feel fatigue or boredom. Because the tasks are small and independent, workers can stop at any time and need not complete all of the tasks for a single policy: subsequent workers can be given tasks that continue at the point at which previous workers stopped working. The small tasks also allow us to better distribute the risk of low-performing crowd workers and the associated costs.

\subsection{Crowd Worker Microtasks}

Steps 3 and 4 are crowd worker microtasks that ask workers to annotate phrases in one of two ways: for step 3, workers are asked to label action verbs that describe information collection, use, transfer, or retention, as shown in Figure 2. Following simple instructions, workers see the $\sim 120$-word paragraph and are tasked to select and annotate relevant phrases using their mouse and keyboard. The annotated phrases are color coded to correspond to the label selected by the worker. The microtask for step 4 is similar, except that, instead of distinguishing among four kinds of actions, workers are asked to identify noun phrases that correspond to any kind of information. In both steps 3 and 4, the results are captured and recorded as part of an AMT batch result, wherein we asked five workers to annotate each paragraph. This number of workers was determined in prior work, which showed worker agreement for $2 / 5$ workers correlates with high precision and recall for these tasks [Breaux and Schaub 2014].

As shown in Figure 1, the results from steps 3 and 4 are combined with a dependency parse of the paragraphs to select action-information pairs in steps A, B1, and B2, which we now discuss.

\subsection{Dependency Parsing and Pair Selection}

In step A, we apply typed dependency parsing to the individual sentences from the microtask text input using the Stanford dependency parser [de Marneffe et al. 2006]. Typed dependencies are binary relations between a first term, called the governor, and a second term, called the dependent. We present an example sentence with the corresponding collapsed, CC-processed dependencies (collapsed dependencies with 
Click here to read the expanded instructions with an example.

Short Instructions: Select the action verbs with your mouse cursor and then press one of the following keys to indicate when the verb describes an act to:

- Press 'c' for collect - any act by Zynga to collect information from another party, including the user

- Press 'u' for use - any act by Zynga or another party to use or modify information for a particular purpose

- Press 't' for transfer - any act by Zynga to transfer or share information with another party, including the user

- Press ' $r$ ' for retain - any act by Zynga to retain, store or delete information

In the following paragraph, any pronouns "We" or "Us" refer to the game company Zynga, and "you" refers to the Zynga user.

\section{Paragraph:}

We may collect or receive information from other sources including (i) other Zynga users who choose to upload their email contacts; and (ii) third party information providers.

Submit Query

Clear Last Clear All

Fig. 2. Crowd worker microtask to annotate information actions.

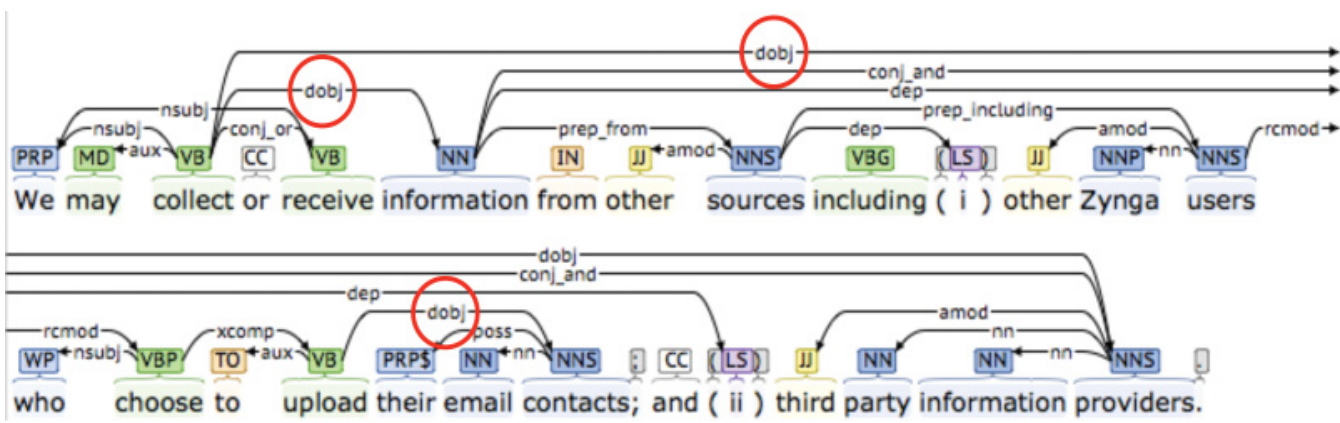

Fig. 3. Stanford dependency parse of microtask input text.

propagation of conjunct dependencies) for each word in the sentence in Figure 3. Commonly found dependency types include $n s u b j$, which is the nominal subject of the sentence, and dobj or direct object of a verb phrase. One advantage of dependency parsing is that the parser splits phrases along conjunctions and links modifiers to nouns. However, natural language ambiguity can lead to errors in parsing. For example, Figure 3 presents three dependences dobj(collect, providers), dobj(collect, information), and dobj(upload, contacts).

The first dependency dobj(collect, providers) is incorrect: the sentence author likely did not mean that the website "collects third-party information providers"; rather, the providers are a second example of "from whom" that information is collected. Thus, we assume some degree of inaccuracy produced by the typed dependency parser. However, the second two dependencies are correct, and indicate prospective goals about which we can ask additional questions: "from whom is information collected" (a collection goal), and "by whom are contacts uploaded" (a transfer goal). Our approach to select action-information type pairs is limited by the accuracy of the Stanford Parser.

We propose two different approaches, denoted by steps B1 and B2, to select the action-information type pairs using the typed dependencies. The typed dependencies are combined with crowd worker annotations in step B2, wherein we perform 
action-information pair selection to identify actions (typically verbs) that should be paired with information types (typically noun phrases). Step B2 is a semiautomated approach that requires manual annotations for the actions and information types, which are obtained from the crowd workers.

In order to automate this process of obtaining the action and information types, we propose an alternate approach in step B1, which is a fully automated approach. In this step, we use the action and information type lexicon, to identify actions and information types in the policy statements using a simple keyword match between the lexicon and policy terms. We combine these identified actions and information types in each statement with the typed dependencies of the statement to determine if the action and information type are linked by a typed dependency or not. If linked, the corresponding action-information type pair is selected as a candidate partial goal specification. We use two general strategies for both steps B1 and B2 for linking action-information pairs: (1) we first identify direct dependencies, in which both the governor and dependent were separately annotated by either the lexicon for B1 or by crowd workers for B2 in the action and information type tasks; and (2) we identify indirect dependencies that consist of two typed dependencies, each one containing one lexicon- or workerannotated term and sharing a third term, which may not have been annotated. We consider only terms that have been annotated by the lexicon in step B1 or by two or more crowd workers in step B2 based on prior work that shows $2 / 5$ workers produce high precision and recall for these tasks [Breaux and Schaub 2014]. In Figure 3, for example, dobj(upload, contacts) is a direct dependency if "upload" was annotated by two or more workers in the action task and "contacts" was annotated by two or more workers in the information type task. In addition, in Figure 3, dobj(collect, information) and cc_or(collect, receive) comprise an indirect dependency that links receive to information via the $c c \_o r$ typed dependency for the English conjunction "or." In our evaluation, we are interested in identifying which dependency types are high confidence, meaning that they maximize true positives and minimize false positives.

Next, we introduce the lexicon as a means to collect and reuse knowledge about annotated actions and information types to improve recall (missing true positives in step B2) and to develop the fully automated approach for step B1.

\subsection{Reusable Lexicon and Entity Extraction}

Lexicons are used to bootstrap requirements analysis by reusing terms frequently seen in particular domains. In our work, we build the lexicon using crowd worker annotations from steps 3 and 4 in Figure 1 for 30 privacy policies to attempt fully automated goal finding. The lexicon is constructed from action and information type entities, which are unique textual descriptions needed to identify recurring instances of the same concept. For instance, the entities in the lexicon should enable us to resolve synonyms, plurals, and singular forms of information types (e.g., "email address" is basically the same concept as "email addresses"). In steps E1 and E2, we apply an entity extraction technique on the annotated verb and noun phrases provided by the crowd workers to extract the individual entities (information types) from the annotated phrases. These phrases may consist of ambiguous lists and clauses that obfuscate the unique entities. The entity extractor was first evaluated on 3,850 crowd worker information type annotations [Bhatia and Breaux 2015]. In Section 4.4, we present an extended evaluation on 7,682 annotations from 30 policies and results of applying the acquired lexicon to the recomposition framework.

The entity extractor workflow is presented in Figure 4. The extractor first tests whether a worker annotation is a list (i.e., it contains a common list delimiter, such as a comma, semicolon or POS-tagged English conjunction CC). If an annotation does not contain a list delimiter, then we test whether the annotation describes a single entity by 


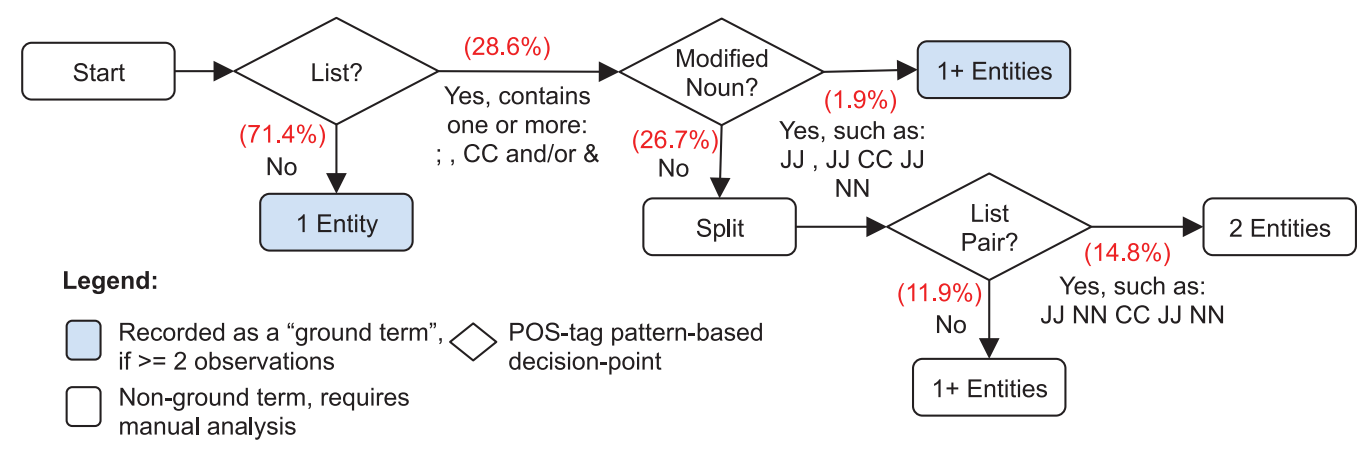

Fig. 4. Workflow for the information entity extractor.

checking the annotation's POS tag sequence against a well-known regular expression $\mathrm{NP}+\mathrm{CL}$ that matches a noun phrase (NP) followed by a clause (CL) expressed as standard POS tags ${ }^{2}$ as follows [Justeson and Katz 1995]:

$$
\begin{aligned}
& \mathrm{NP}=((\mathrm{JJ}|\mathrm{RB}| \mathrm{VBG}|\mathrm{VBD}| \mathrm{NN} \backslash \mathrm{S} ? \mid \mathrm{NN} \backslash \mathrm{S} ? \backslash \mathrm{SPOS}) \backslash \mathrm{s})^{*}(\mathrm{NN} \backslash \mathrm{S} ?) \\
& \mathrm{CL}=\left(\backslash \mathrm{s}(\mathrm{IN}|\mathrm{PRP}| \mathrm{TO}|\mathrm{VBG}| \mathrm{VBN}|\mathrm{WDT}| \mathrm{WP}) \backslash \mathrm{s}^{*}\right) ?
\end{aligned}
$$

Based on our analysis of 30 policies, $71.4 \%$ of the worker-supplied information type annotations describe single entities, and the remaining $28.6 \%$ describe lists. For lists, the extractor checks whether the annotation describes a modified noun, which comprises $1.9 \%$ of annotations. This case includes lists of conjoint adjectives followed by a noun (e.g., "aggregate, statistical information"), as well as disjoint lists (e.g., "geographic and demographic information"). Disjoint lists are split to distribute the modifiers separately across the nouns (e.g., to yield "geographic information" and "demographic information"). The remaining $26.7 \%$ of annotations are lists of noun phrases, which are split by delimiter. Each delimiter-separated noun phrase is checked against previously seen simple, nonobfuscated entities, called ground terms. In Figure 4, ground terms are automatically identified where the output boxes are colored blue. While the workflow is seemingly complex, it has been shown to be highly effective at extracting entities, as we discuss in Section 4.4.

We next discuss the crowd worker pair-validation task in step 5.

\subsection{Validate Pairs and Identify Source and Target}

In step 5, we take the selected action and information type pairs from step B2 and send these pairs to the crowd workers to ask whether the information action and information type are valid pairs. If true, we also ask crowd workers to identify the actors who send, receive, and use the information based on the coded action type. This validation task helps us to remove the false-positive action-information type pairs produced by step B2 because, unlike the crowd workers who understand context, the lexicon indiscriminately identifies all candidate pairs based on keyword matches. Figure 5 presents the task interface for step 5, in which workers select the action modality ("permits" or "prohibits"), the action category, and then they complete the source and target questions using radio buttons or free-response text boxes. If the worker selects collection from the drop-down list, the questions ask "from whom," whereas selecting transfer

\footnotetext{
${ }^{2}$ IN: Preposition or subordinating conjunction; JJ: Adjective; NN: Noun; POS: Possessive ending; PRP: Preposition; TO: to; RB: Adverb; VBD: Verb; past tense: VBG Verb, gerund or present participle, VBN Verb, past participle.
} 


\section{Click here to show the instructions.}

In the following statement, any pronouns "We" or "Us" refer to the company Zynga and "you" refers to the Zynga user.

Statement: We may collect or receive information from other sources including (i) other Zynga users who choose to upload their email contacts; and (ii) third party information providers.

Is the highlighted information type (blue) being acted upon by the action (orange)? $\odot$ Yes, $\bigcirc$ No.

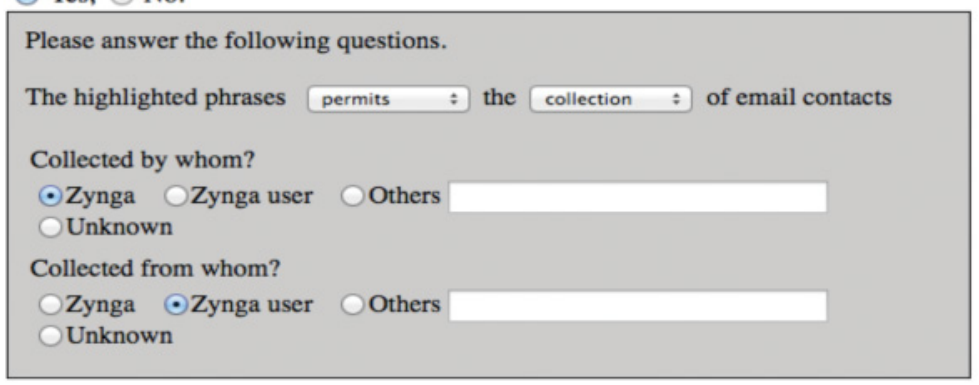

Submit Query

Fig. 5. Crowd worker microtask to validate action-information type pairs.

from the drop-down list asks "to whom." For use and retention, we ask only "by whom" is the information used or retained.

Our framework makes use of crowd worker annotations to identify the actions and information types in the privacy policy statements, which are linked using the typed dependencies to select the action-information type pairs for each statement. The action and information type annotations are used to build the action and information type lexicon, respectively, which are reused to identify missing crowd worker annotations, and to attempt to fully automate the crowd worker annotation process. We next present the results of our framework evaluation.

\section{EVALUATION AND RESULTS}

The task recomposition framework presented in Section 3 combines human annotation with natural language dependency parsing and a reusable lexicon to identify actioninformation type pairs that comprise partial specifications of data processing.

We evaluated the framework by answering the following research questions:

RQ1. How do crowd workers compare with expert annotators in performing microtasks?

RQ2. How well do typed dependencies combined with crowd worker annotations predict the pairs needed to express partial goal specifications?

RQ3. How well does the lexicon improve identification of missing annotations or pairs?

RQ4. How well does lexical reuse increase with each new policy analyzed?

RQ5. How well do crowd workers identify false positives in a validation task?

RQ1 evaluates steps 3 and 4 in the framework (see Figure 1) with respect to precision and recall using the expert annotations. This evaluation extends a prior evaluation of these two tasks that examined only a single policy [Breaux and Schaub 2014]. RQ2 evaluates the typed dependency step A and pair selection step B2 against the 
expert pairs, while RQ3 separately evaluates pair selection step B1 using the crowd worker annotations and lexicon against the expert pairs. RQ4 evaluates the lexicon independently to assess how it scales over time. Finally, RQ5 evaluates step 5 and the ability of the crowd workers to identify false pairs against the expert pairs.

To evaluate our hybrid framework and to answer the research questions, we selected five privacy policies that the first two authors (the experts) analyzed as part of this case study, which we refer to as expert annotations and expert pairs when combined into a partial goal specification:

- AOL Advertising, last updated May 4, 2011

- Facebook API Developer Guidelines, revised June 28, 2013

- Flurry Privacy Policy, updated July 9, 2013

- Waze Privacy Policy, modified May 30, 2013

- Zynga Privacy Policy, last updated September 30, 2011

These policies were selected because they were used in two prior case studies to express privacy goals formally in the Eddy language based on Description Logic [Breaux et al. 2014, 2015]. The policies correspond to different stakeholders in a software composition: the AOL and Flurry policies govern advertising services used by a game provider (Zygna) and a navigation application (Waze). The Facebook policy governs a platform that both Zynga and Waze use for user-identification services, when users log in to their applications using their Facebook accounts. Thus, these policies cover two popular dataflows and the policy language in each of these policies varies by the role of the covered services (ad services, identity provider, and first-party app developers).

The expert dataset was created by two analysts (the first and second authors) by extending the annotations from a prior case study [Breaux et al. 2014]. In this prior study, on average, the first analyst expended $1.09 \mathrm{~min}$ per statement extracting requirements, whereas the second analyst expended $2.21 \mathrm{~min}$ per statement [Breaux et al. 2014]. For the new dataset, the analysts spent on average $1.9 \mathrm{~min}$ each per statement to review the previous annotations and extend the dataset. The expert dataset serves as the "ground truth" by which we compute precision and recall as measures of the automated steps B1 and B2 shown in Figure 1. For all precision and recall calculations, the expert dataset contains the sum of true positives and false negatives.

We now discuss our empirical results with respect to each research question.

\subsection{Crowd Worker Microtask Results}

We solicited five workers per microtask to identify the actions and information types. We recruited US residents as workers on AMT, who had at least a 95\% approval rating for over 5,000 tasks. We paid workers $\$ 0.15$ per task for actions and $\$ 0.12$ per task for information types to keep the hourly wage close to $\$ 8$ to $\$ 10$ per hour. We allowed up to 5 min to complete each task. Results were accepted or rejected within 24 hours. For the action identification task, the workers required $72 \mathrm{~s}$, on average, to complete a single task, which resulted in an average hourly rate of $\$ 8.40$. On average, workers required 61 s per information type task, with an average hourly rate of $\$ 6.30$.

Table I presents the total cost incurred for the information action and information type identification microtasks for all policies, including the total number of tasks (Tasks) in each policy; Amazon charges of 10\% (AMT fees), and Total Cost, consisting of worker payments and AMT fees.

Table II presents the number of annotations acquired from steps 3 and 4: for each policy, we present the total number of sentences in the policy, the total number of sentences with annotated actions only, with annotated information types only, with both an annotated action and information type, and, finally, the overall total number of annotated actions and information types. For sentences with only the annotated 
Table I. Cost to Crowdsource the Microtasks

\begin{tabular}{|l|c|c|c|c|c|c|}
\hline \multirow{2}{*}{ Policy } & \multicolumn{3}{|c|}{ Actions Microtask } & \multicolumn{3}{c|}{ Info. Types Microtask } \\
\cline { 2 - 7 } & Tasks & AMT fees & Total Cost & Tasks & AMT fees & Total Cost \\
\hline Waze & 34 & $\$ 2.55$ & $\$ 28.05$ & 34 & $\$ 2.04$ & $\$ 22.44$ \\
\hline Zynga & 32 & $\$ 2.40$ & $\$ 26.40$ & 32 & $\$ 1.92$ & $\$ 21.12$ \\
\hline Flurry & 33 & $\$ 2.48$ & $\$ 27.23$ & 33 & $\$ 1.98$ & $\$ 21.78$ \\
\hline FB & 32 & $\$ 2.40$ & $\$ 26.40$ & 32 & $\$ 1.92$ & $\$ 21.12$ \\
\hline AOL & 18 & $\$ 1.35$ & $\$ 14.85$ & 18 & $\$ 1.08$ & $\$ 11.88$ \\
\hline
\end{tabular}

Table II. Summary of Microtask Annotations

\begin{tabular}{|l|c|c|c|c|c|c|}
\hline & & \multicolumn{3}{|c|}{ Sentences with: } & \multicolumn{2}{c|}{ Annotations } \\
\cline { 3 - 7 } Policy & Total Sentences & Only Actions & Only Info Types & Both & Actions & Info Types \\
\hline Waze & 117 & 5 & 36 & 56 & 117 & 146 \\
\hline Zynga & 97 & 4 & 28 & 52 & 103 & 125 \\
\hline Flurry & 135 & 22 & 32 & 49 & 106 & 111 \\
\hline FB & 136 & 15 & 25 & 57 & 129 & 166 \\
\hline AOL & 76 & 6 & 6 & 50 & 96 & 87 \\
\hline
\end{tabular}

Table III. Crowdsourced Action and Information Type Annotations Compared to Expert

\begin{tabular}{|l|c|c|c|c|}
\hline \multirow{2}{*}{ Policy } & \multicolumn{2}{|c|}{ Actions } & \multicolumn{2}{c|}{ Information Types } \\
\cline { 2 - 5 } & Precision & Recall & Precision & Recall \\
\hline Waze & 0.88 & 0.83 & 0.62 & 0.91 \\
\hline Zynga & 0.91 & 0.79 & 0.95 & 0.98 \\
\hline Flurry & 0.73 & 0.64 & 0.97 & 0.84 \\
\hline FB & 0.98 & 0.96 & 0.88 & 0.90 \\
\hline AOL & 0.86 & 0.98 & 0.71 & 0.95 \\
\hline Average & 0.87 & 0.84 & 0.83 & 0.92 \\
\hline
\end{tabular}

actions or information types and not both, these sentences would not yield an actioninformation type pair based on an expert analysis of the text.

Table III presents the precision and recall for both actions and information types as compared to the expert annotations. On average, workers were able to identify the actions and information types with high recall of 0.84 and 0.92 , respectively, and average precision of 0.87 and 0.83 , respectively. Notable in Table III, the Flurry policy includes nomenclature specific to the advertising industry that crowd workers are likely unfamiliar with, which may explain the lower precision and recall for that policy as compared to the other policies.

\subsection{Dependency Parse and Pair Selection Results}

We now present the dependencies parser results and results of our techniques for selecting action-information type pairs.

Table IV presents results from a naïve approach to produce typed dependencies from the five policies to illustrate the scope of the pair selection challenge. This includes the number of unfiltered dependencies per policy (Total Dependencies); the subset of the total in which the governor or dependent are a verb and noun pair (Dependencies $w / V e r b s \&$ Nouns); the three most common dependency types found in the direct selection method described in Section 3.2, which are dobj (direct object of a verb phrase), nsubjpass (syntactic subject of a passive clause) and vmod (verb heading a phrase); and the number of pairs identified in the expert analysis, which represents our evaluation target. As can be seen from Table IV, the space of dependencies is quite large and, assuming perfect recall, the precision of a naïve approach to pair selection would be very low. 
Table IV. Naïve Approach to Identify Relevant Pairs-Parser

\begin{tabular}{|l|c|c|c|c|}
\hline Policy & $\begin{array}{c}\text { Total } \\
\text { Dependencies }\end{array}$ & $\begin{array}{c}\text { Dependencies } \\
\text { w/Verbs \& Nouns }\end{array}$ & $\begin{array}{c}\text { dobj, nsubjpass, } \\
\text { vmod }\end{array}$ & $\begin{array}{c}\text { Expert } \\
\text { Pairs }\end{array}$ \\
\hline Waze & 3286 & 794 & 365 & 101 \\
\hline Zynga & 2758 & 655 & 352 & 93 \\
\hline Flurry & 3268 & 845 & 398 & 81 \\
\hline FB & 3389 & 765 & 339 & 91 \\
\hline AOL & 1720 & 452 & 216 & 81 \\
\hline
\end{tabular}

Table V. Naïve Approach to Identify Relevant Pairs-Parser and Lexicon

\begin{tabular}{|l|c|c|c|c|}
\hline Policy & Expert Pairs & Lexicon and Parser Pairs & Precision & Recall \\
\hline Waze & 101 & 360 & 0.22 & 0.77 \\
\hline Zynga & 93 & 424 & 0.19 & 0.86 \\
\hline Flurry & 81 & 432 & 0.15 & 0.79 \\
\hline FB & 91 & 306 & 0.22 & 0.75 \\
\hline AOL & 81 & 229 & 0.22 & 0.79 \\
\hline
\end{tabular}

In Table $\mathrm{V}$, we present a slightly more informed approach to identify action and information type pairs using typed dependencies and lexicon (B1 in Figure 1). The column Expert Pairs lists the total number of action and information type pairs identified by the experts manually. The column Lexicon and Parser Pairs lists the total number of pairs automatically obtained by pairing actions and information types from the lexicons that share a direct or indirect dependency based on the parser output. The columns Precision and Recall are computed by comparing the Lexical and Parser Pairs to the Expert Pairs, which serves as the ground truth. The lexicon-based approach was able to identify the action-information type pairs with an average recall of 0.80 ; however, the average precision was very low at 0.20 . The large number of false positives obtained using the lexicon can be attributed to the fact that, at present, the lexicon does not have the ability to disambiguate the meaning of a term in the given context, thus identifies all instances of a term in a statement.

From Tables IV and V, we see that semantic dependencies alone, even direct dependencies without human guidance, produce a large number of false positives compared to the evaluation target. In addition, while the lexicon contains terminology from prior worker annotations, it lacks the workers' direction in reducing the dependencies to within a reasonable reach of the evaluation target. To inform our approach, we analyzed the direct and indirect dependencies to determine the most frequent dependency patterns found in the recombinations and how often they lead to true or false positives. We found three direct dependency patterns and five indirect dependency patterns that constitute $71.81 \%$ of the total true positive recombinations. We describe these patterns in Table VI as follows: the pattern name, the typed dependency sequence, the frequency of the pattern across all five policies, and the number of true- and false-positive action-information type pairs for each pattern measured against the expert pairs.

The three direct dependency patterns (direct object, passive nominal subject, and verbal modifier) in Table VI constitute, on average, $59.1 \%, 10.3 \%$, and $7.3 \%$, respectively, of the direct dependency recompositions across all five policies for the hybrid approach. These three patterns led to true positives in $99.6 \%$ of the instances studied. The only instance in which the direct object pattern yields an incorrect result was "You must immediately revoke an end-advertiser's access to your app upon our request" (from Facebook privacy policy). In this sentence, revoke is annotated as an information action and access is annotated as an information type by the workers. The pair (revokeaccess) is linked by a direct object dependency, which is a true dependency yet a false positive because "access" is not an information type. 
Table VI. Typed Dependency Patterns

\begin{tabular}{|c|c|c|c|c|c|}
\hline & Pattern Name & $\begin{array}{c}\text { Typed Dependency } \\
\text { Sequence }\end{array}$ & Frequency & $\begin{array}{c}\text { True } \\
\text { Positive }\end{array}$ & $\begin{array}{c}\text { False } \\
\text { Positive }\end{array}$ \\
\hline \multirow{3}{*}{ 苍 } & Direct Object & dobj & 195 & 188 & 7 \\
\hline & Passive nominal subject & nsubjpass & 34 & 32 & 2 \\
\hline & Verbal modifier & vmod & 24 & 22 & 2 \\
\hline \multirow{5}{*}{ 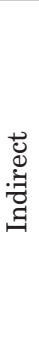 } & $\begin{array}{l}\text { Conjunction and with } \\
\text { direct object }\end{array}$ & conj_and, dobj & 25 & 15 & 10 \\
\hline & $\begin{array}{l}\text { Conjunction or with } \\
\text { direct object }\end{array}$ & conj_or, dobj & 17 & 12 & 5 \\
\hline & $\begin{array}{l}\text { Passive nominal subject } \\
\text { with list }\end{array}$ & nsubjpass, prep_such as & 1 & 1 & 0 \\
\hline & $\begin{array}{l}\text { Direct object with verbal } \\
\text { modifier }\end{array}$ & dobj, vmod & 13 & 2 & 11 \\
\hline & $\begin{array}{l}\text { Direct object with } \\
\text { preposition }\end{array}$ & dobj, prep_* & 20 & 10 & 10 \\
\hline
\end{tabular}

Table VII. Action-Information Type Pairs from Hybrid Approach

\begin{tabular}{|l|c|c|c|c|c|}
\hline Policy & $\begin{array}{c}\text { Total Annotated } \\
\text { Pairs, Possible }\end{array}$ & $\begin{array}{c}\text { High-Confidence } \\
\text { Pairs }\end{array}$ & $\begin{array}{c}\text { Expert } \\
\text { Pairs }\end{array}$ & Precision & Recall \\
\hline Waze & 379 & 107 & 101 & 0.73 & 0.77 \\
\hline Zynga & 467 & 120 & 93 & 0.64 & 0.83 \\
\hline Flurry & 237 & 71 & 81 & 0.79 & 0.69 \\
\hline FB & 301 & 111 & 91 & 0.75 & 0.91 \\
\hline AOL & 239 & 106 & 81 & 0.74 & 0.96 \\
\hline
\end{tabular}

The five indirect dependency patterns describe $41.1 \%$ of the total indirect dependency recompositions in the hybrid approach action and information type pairs. On average, the direct dependency patterns led to true positives in $87.9 \%$ and indirect dependency patterns led to true positives in only $44.3 \%$ of the instances.

As observed from Tables IV and V, there is no simple approach to using the parser to identify the action-information type pairs. By adding our crowd worker annotations for both the actions and information types, however, we identified a set of high-confidence pairs that consist of the direct and indirect pairs defined in Section 3.2. Ideally, these pairs will contain all true positives and minimal false positives, and omit minimal false negatives. In Table VII, we present our baseline measure (Total Annotated Pairs), which is the number of all possible pairs, which assumes naïvely that every annotated information action is crossed with every information type that occurs in the same sentence, followed by the total number of high-confidence pairs based on dependency parsing and worker annotations, and the total number of expert pairs. The hybrid approach greatly reduces the number of pairs as compared to the naïve approaches presented in Tables IV and V.

In Table VII, the Flurry policy has the lowest precision and recall among all analyzed policies. This is because workers annotated both the information action and information type in only $36.3 \%$ of the sentences in the Flurry policy (see Table II), whereas in other policies, workers annotated $52.3 \%$, on average. The actions in the Flurry policy that were not identified by the workers were context-sensitive-for example, "get back" (a colloquialism), and "export" and "request" (both software functions), to name a fewwhich were also different from the action words frequently found in other policies. Thus, the workers biased by terminology commonly found in other policies may have not expected, thus missed, these terms.

Our analysis of the remaining 143 false-positive pairs after the expert analysis shows that 14/143 pairs contain an action that was part of a data purpose. For example, in the 
Table VIII. Results for Reusable Lexicon

\begin{tabular}{|l|c|c|c|}
\hline Policy & $\begin{array}{c}\text { New Actions, } \\
\text { Missed }\end{array}$ & $\begin{array}{c}\text { New Information } \\
\text { Types, Missed }\end{array}$ & $\begin{array}{c}\text { New High-Confidence } \\
\text { Pairs, Missed }\end{array}$ \\
\hline Waze & 116 & 17 & 58 \\
\hline Zynga & 165 & 19 & 74 \\
\hline Flurry & 88 & 99 & 78 \\
\hline FB & 81 & 79 & 51 \\
\hline AOL & 20 & 36 & 26 \\
\hline
\end{tabular}

sentence "We use personal information to create new services," the action "create" marks the beginning of the purpose for which the information type personal information is being used. We observed that 12/143 pairs were pairs in which a technology was being used to perform an information action. For example, "This information is collected by the use of log-files." In this case, the log-files are a container for information and a technology. Manually excluding such pairs from our analysis would improve average precision from 0.73 to 0.78 , which offers promise for future work.

In addition, we manually analyzed the 75 false-negative sentences from all five policies in which the action and information type pairs were identified by the experts, but were missed by our crowd workers. Our analysis shows that, out of the 75 sentences, the workers did not annotate an information action in 37/75 of these sentences; in 20/75 of these sentences, the workers did not annotate an information type; and in 5/75, the workers did not annotate both the action and the information type. In Section 4.3, we discuss how we make use of the reusable lexicon to identify these missing annotations and reduce the number of false negatives.

In the remaining 13/75 sentences, the workers had identified the information action and the information type, but the parser could not determine a direct or indirect dependency between the information action and information type in the pairs; thus, they were not included in our high-confidence pairs. On further inspection, we found that this was because of incomplete worker annotations. For example, in the sentence, "You can retrieve recommendations created for a particular End User by passing the device identifier of the End User," the workers annotated the information action retrieved but missed its corresponding information type (recommendations). Instead, they annotated the information type device identifier, but missed its corresponding information action (passing). The annotated information action and type pair (retrieved-device identifier) is not linked by a direct or indirect dependency relationship, thus was excluded from the high-confidence pairs.

In the next section, we discuss the reusable lexicon's impact on identifying missing actions and information types.

\subsection{Impact of Reusable Lexicon on Pair Selection}

We now present the results of the reusable lexicon. We built the lexicon from 30 policies spanning five domains: employment, news, social networking, shopping, and telecommunications. The five policies listed in the beginning of Section 4 were not part of the selected policies. The entity extractor successfully extracted entities from $97.8 \%$ of crowd worker annotations. In Table VIII, we present the number of actions and information types that were missing from the crowd worker annotations and identified using the lexicon, and the corresponding number of missing high-confidence direct and indirect pairs that result from applying the lexicon to each of the five policies that are used for evaluation.

Table IX presents the number of false-negative pairs produced from worker annotations reported in Section 4.2, the number of true-positive pairs identified using the high-confidence pairs from the lexicon reuse reported in Table VIII, the precision and 
Table IX. Impact of Lexical Reuse on Precision and Recall

\begin{tabular}{|l|c|c|c|c|c|c|}
\hline Policy & $\begin{array}{c}\text { False-Negative } \\
\text { Pairs }\end{array}$ & $\begin{array}{c}\text { True-Positive } \\
\text { Pairs }\end{array}$ & $\begin{array}{c}\text { Precision } \\
\text { w/o Lexicon }\end{array}$ & $\begin{array}{c}\text { Precision } \\
\text { w/Lexicon }\end{array}$ & $\begin{array}{c}\text { Recall } \\
\text { w/o Lexicon }\end{array}$ & $\begin{array}{c}\text { Recall } \\
\text { w/Lexicon }\end{array}$ \\
\hline Waze & 23 & 6 & 0.73 & 0.51 & 0.77 & 0.83 \\
\hline Zynga & 16 & 6 & 0.64 & 0.43 & 0.83 & 0.92 \\
\hline Flurry & 25 & 12 & 0.79 & 0.46 & 0.69 & 0.84 \\
\hline FB & 8 & 3 & 0.75 & 0.52 & 0.91 & 0.95 \\
\hline AOL & 3 & 1 & 0.74 & 0.60 & 0.96 & 0.98 \\
\hline
\end{tabular}

Table X. Impact of Lexical Reuse on Precision and Recall

\begin{tabular}{|l|c|c|c|c|c|c|}
\hline \multirow{2}{*}{ Action Lexicon } & \multicolumn{2}{|c|}{ 10\% Info. Type Lexicon } & \multicolumn{2}{c|}{ 50\% Info. Type Lexicon } & \multicolumn{2}{c|}{ 100\% Info. Type Lexicon } \\
\cline { 2 - 7 } & Precision & Recall & Precision & Recall & Precision & Recall \\
\hline 10\% Action Lexicon & 0.63 & 0.88 & 0.61 & 0.89 & 0.60 & 0.89 \\
\hline $50 \%$ Action Lexicon & 0.56 & 0.89 & 0.54 & 0.90 & 0.53 & 0.90 \\
\hline $100 \%$ Action Lexicon & 0.53 & 0.90 & 0.52 & 0.90 & 0.51 & 0.91 \\
\hline
\end{tabular}

recall without the lexicon reported in Table VII, and the precision and recall with the lexicon. The results in Tables VIII and IX were computed using all the terms in the action lexicon and information type lexicon.

The lexicon-produced high-confidence pairs identified $37.34 \%$ of the pairs that were FNs from the worker annotations and improved the average recall by $8.8 \%$ to 0.90 . However, the lexicon also significantly reduces the average precision by $31 \%$ to 0.50 based on the expert pairs. As has been previously noted by Berry et al. [2012], however, it is difficult to achieve both high precision and high recall with NLP techniques for requirements engineering, and an NLP tool for requirements engineering should be tuned to favor recall over precision because errors of commission are generally easier to correct than errors of omission. We therefore aim at minimizing the number of false negatives, even if that means accepting a few false positives.

When using all the terms from the action and information type lexicons, precision drops by $31 \%$ for an $8.8 \%$ increase in the recall over the hybrid pair results in Table VII. This decrease in precision is due to the effect of context in terminological reuse. Phrases such as "send" or "receive" may indicate information collection and transfer in one context, but may be used to describe noninformation transactions in another context. To find the optimal subset of the lexicons that leads to an increase in recall without a steep decrease in the precision, we conducted an experiment based on lexicon partitions portioned from increasing increments of $10 \%$. In Table X, we present the Precision and Recall for different lexicon partitions. The column Action Lexicon shows the partition of the action lexicon that was used for the respective experiment. In Table $\mathrm{X}, x \%$ Action Lexicon means that the top $\mathrm{x}$ percent of the terms in the action lexicon were used for the analysis. Similarly, X\% Info. Type Lexicon means that the top x percent of the terms in the information type lexicon were used for the analysis.

From our experiments with the lexicon partitions, we conclude that the precision decreases and recall increases as the partition size of the lexicon for the experiment increases. Further, the decrease in precision is greater than the increase in recall. The precision drops by $14.3 \%$ and the recall increases by $5.4 \%$ over the hybrid pair results in Table VII when we use the top $10 \%$ terms in the action and information type lexicons. The precision further decreases as we increase the partition sizes and the precision drops by $31 \%$ when the entire action and information type lexicons are used, for an increase of $8.8 \%$ increase in recall.

Even after lexical reuse, some information actions from the expert annotations could not be identified using the hybrid approach and lexical reuse. These actions include "based on," "get back," "complete" (a user profile), and "be visible," which are contextsensitive or require rich interpretations, such as multiple inferences or tacit knowledge 


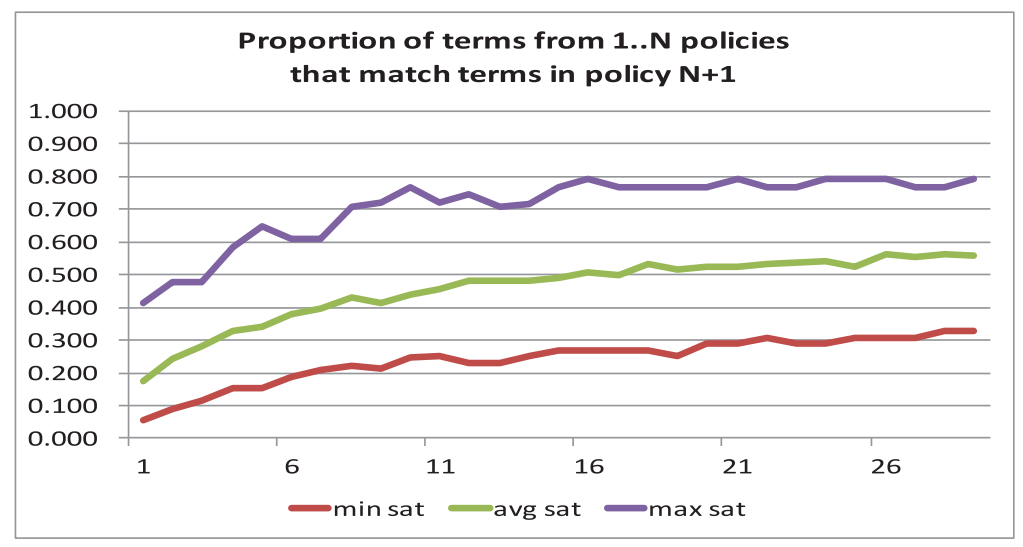

Fig. 6. Saturation of information types in lexicon: we observe the percentage reuse of information types described in N-1 policies for each Nth policy along the x-axis.

(e.g., "be visible" suggests that others can see the information that has been visible; this inference constitutes a form of information transfer). The lexicon is missing some information types, which include domain-specific information not present in the lexicon: for example, "customized audience" and "identifiable-route." Missed information types also include anaphora, such as "it," that refer to an information type in the prior sentence, which was identified by the experts, but not by the workers.

The first and third authors of this article evaluated the lexicon to determine the scale of false positives that the lexicon can introduce when used without worker annotations. They analyzed the Waze and Zynga policies to identify those instances of actions and information types that appear in the lexicon, but were not annotated by the workers (i.e., to find possible false negatives). They identified 909 actions and 450 information types in the two policies, among which only $15 \%$ of the actions and $12.2 \%$ of the information types were false negatives. From this analysis, we conclude that worker ability to distinguish between true positives and false positives is an improvement over the lexicon alone, and the lexicon alone could greatly inflate the number of false positives, if used without worker annotations.

In summary, the low precision due to the lexicon can be attributed, in part, to the ambiguity of terms and the role of context in determining when data processing events take place, as well as to the noise in worker responses. Information actions that are ambiguous include "assist," "solicit," "permit," and "allow," among others. Terms that workers annotated as information types that should be excluded include "third parties," "campaign," and "network." In the case of "campaign" and "network," these are activities and technologies that imply some type of information, but are not themselves the implied information type. We also observed that false negatives in the worker data include words that occur less frequently across policies, including actions, such as "permit" and "export," and information types, such as "payment" and "ads." Thus, limiting the lexicon to the most frequent words and phrases will, in turn, hinder the ability of the lexicon to identify false negatives.

\subsection{Results of Scaling Reusable Lexicon}

We examined the extent to which the lexicon can predict actions and information types in additional privacy policies. This analysis shows that privacy policies have unique entities that are not shared across policies. Figure 6 presents the saturation (sat.) of information type entities for the same 30 policies: at any point along the $\mathrm{x}$-axis, we 


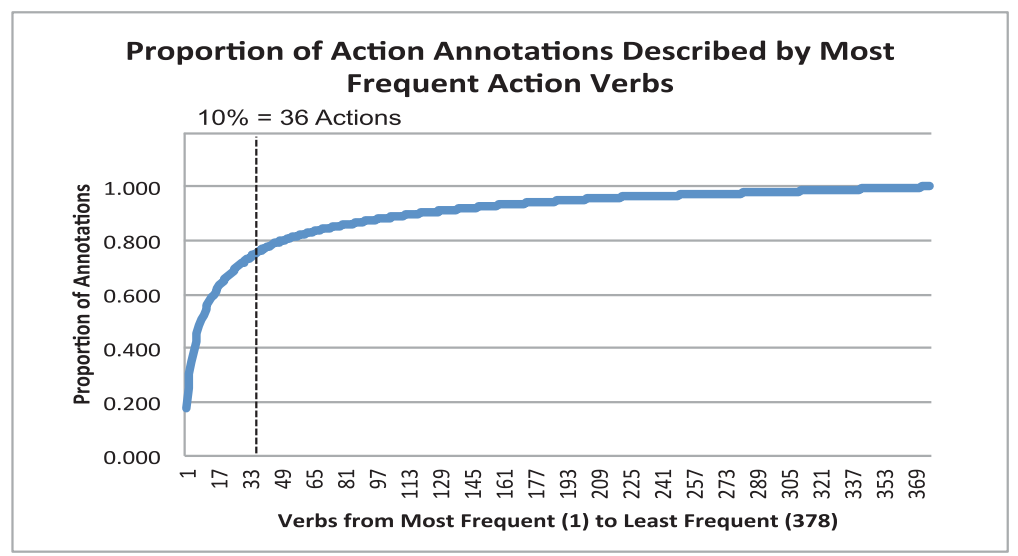

Fig. 7. Number of annotations per verb along the y-axis (log scale), and each unique verb of 380 verbs along $\mathrm{x}$-axis; $10 \%$ of verbs covered $75 \%$ of annotations.

observe the percent reuse of information types in a policy $\mathrm{N}$ based on the last $\mathrm{N}-1$ policies previously seen. This result is based on 100 pseudo-random permutations of the orders of the 30 annotated policies. We observe that near 14 to 15 policies, the average maximum threshold for saturation of $77 \%$ is achieved, meaning that every new policy contributes a sufficient number of unique terms to the lexicon that $23 \%$ of the new policy would not appear in any previously seen policy in the best case, and $71 \%$ of the policy terms would be new in the worst case. At present, this observation suggests that the lexicon cannot entirely replace crowd workers, because there will always be new terms that the lexicon has never encountered.

We further analyzed the action verbs from the same 30 policies and found 377 unique verbs identified by crowd workers. Only a small subset of these verbs dominate the results, with $10 \%$ of action verbs describing $75 \%$ of the annotations (see Figure 7 , which shows the number of annotations per verb on the y-axis in logarithmic scale, and each indexed verb along the x-axis). There is ambiguity, however, with $28 \%$ of verbs coded by two or more actions (collect, use, transfer, and retain) and 5\% of verbs coded as sharing-ambiguous, meaning they were coded as both collect and transfer by two or more workers. For these verbs, it may be difficult for crowd workers to determine from the text who is providing and who is receiving the relevant information. Finally, some of the verbs were also used to describe use-related purposes, which is one source of reduced precision discussed in Section 4.3.

\subsection{Validation Task Result}

The results from Section 4.2 show that the high-confidence pairs from our hybrid approach contain some number of false positives (see steps A and B2 in Figure 1, and Tables VII and VIII). One objective of step 5 in our framework (see Figure 1) is to identify these false positives and remove them from the results to achieve higher average precision. In this validation task, we ask workers whether the action and information type pair from the high-confidence pairs is a valid pair (true positive), or whether it is an invalid pair (false positive). If a valid pair, then we ask crowd workers to identify the modality and the actors who send, receive, and use the information based on action labels provided by the previous crowd workers in step 4 .

We solicited five workers per task to identify the valid information actions and information type pairs. We recruited US residents as workers on AMT, who had at least a $95 \%$ approval rating for over 5,000 tasks. We paid workers $\$ 0.12$ per classification 
Table XI. Pairs Validation Result

\begin{tabular}{|l|c|c|c|c|c|c|}
\hline Policy & $\begin{array}{c}\text { High-Confidence } \\
\text { Pairs }\end{array}$ & $\begin{array}{c}\text { False Pairs } \\
\text { by Worker }\end{array}$ & $\begin{array}{c}\text { False Pairs } \\
\text { by Experts }\end{array}$ & $\begin{array}{c}\text { Ambiguous } \\
\text { Pairs }\end{array}$ & $\begin{array}{c}\text { Precision } \\
\text { w/o Validation }\end{array}$ & $\begin{array}{c}\text { Precision } \\
\text { w/Validation }\end{array}$ \\
\hline Waze & 107 & 20 & 30 & 12 & 0.73 & 0.88 \\
\hline Zynga & 120 & 44 & 43 & 13 & 0.64 & 0.94 \\
\hline Flurry & 71 & 11 & 16 & 4 & 0.79 & 0.92 \\
\hline FB & 111 & 27 & 28 & 20 & 0.75 & 0.91 \\
\hline AOL & 106 & 32 & 28 & 17 & 0.74 & 0.99 \\
\hline
\end{tabular}

task. We allowed up to $5 \mathrm{~min}$ to complete the task. Results were accepted or rejected within $24 \mathrm{~h}$. The workers completed each task in $39.6 \mathrm{~s}$, on average, resulting in an average hourly rate of $\$ 10.95$.

In the analysis of the validation task, we mark a pair as false positive if more workers annotated it as false positive than the number of workers who annotated it as true positive. Table XI presents the validation task results as follows: the number of high-confidence pairs obtained using our hybrid approach (see results in Section 4.2 from step B2 in Figure 1); the number of false-positive pairs identified by three or more workers, the number of false-positive pairs identified by the experts; the number of ambiguous pairs, in which $2 / 5$ and $3 / 5$ workers yielded conflicting annotations; the precision without validation reported in Table VII; and the precision with validation from crowd workers. As shown in Table XI, the crowd workers greatly reduced the number of false positives produced by the direct and indirect dependency patterns.

\section{THREATS TO VALIDITY}

In this section, we discuss the threats to construct, internal, and external validity for our framework, which makes use of crowdsourcing and NLP techniques.

\subsection{Construct Validity}

Construct validity describes whether what we proposed to measure is indeed what we measured [Yin 2009]. One concern in our framework is whether crowd workers accurately understood what constitutes an action and information type during the annotation task. To address this concern, we provide detailed instructions and a worked example in the microtask description to help crowd workers understand what kinds of phrases match our interpretation of action and information types (see Section 3.1). Furthermore, we consider only annotations for which two or more crowd workers agreed on the same annotation. We also compare these crowd-worker annotations to expert annotations to measure the extent to which crowd workers agreed with the experts. On average, workers identified actions and information types with high average recall of 0.84 and 0.92 , respectively, and high average precision of 0.87 and 0.83 , respectively, as shown in Table III. Sources of disagreement often arose when a given word or phrase is ambiguous. For example, the action "access" can be annotated as collect, use, or transfer, depending on who is performing the action (the end user, the company, or a third party).

\subsection{Internal Validity}

Internal validity is the extent to which observed causal relationships exist within the data and, particularly, whether the investigator's inferences about the data are valid [Yin 2009]. In our framework, our conclusions depend on the reliable performance of the Stanford Parser to identify typed dependencies. For example, the per-dependency accuracy of the Stanford Parser has been reported to be 80.3\% [de Marneffe et al. 2006]. Changes in the parser accuracy will affect overall performance. In addition, the types of policies that we studied could present potential confounds in the form of selection bias: 
policies with more or less technical information types or policies that describe new, previously unknown technologies, could be harder for workers to annotate, because they may not recognize the associated information types. The task of interpreting natural language text is subjective-even the experts could miss an annotation, or could be influenced by the interpretations discovered by the crowd workers. But, for the purpose of evaluating our approach, we use the annotations generated by the experts as ground truth. We therefore note that this expert dataset contains the true positives and false negatives for our evaluation purposes.

\subsection{External Validity}

External validity is the extent to which our approach generalizes to the population outside the sample used in the study [Yin 2009]. Based on our study of crowd-worker interpretation of the privacy policy text (see results in Table III) and on the Stanford Parser identification of typed dependencies linking annotated words, we believe that our approach could be used for other information-related legal texts. However, we hesitate to make this claim without conducting further studies with different legal text and in other domains. For complex laws, such as the Privacy Rule of the Health Insurance Portability and Accountability Act (HIPAA), there may be additional steps required to preprocess and convert the documents into microtasks for crowdsourcing, which we further discuss in Section 6.

\section{DISCUSSION AND SUMMARY}

We now discuss our hybridized recomposition approach in the light of our results and the potential for future work. The analyses conducted for the five research questions show complementary results. With respect to RQ1, which compares the crowd-worker performance to expert annotators, we find that untrained crowd workers can be used to elicit most of the actions and information types that were identified by the experts, which leads to high recall for the crowdsourcing action and information type microtasks when compared to the expert annotations. Moreover, we discovered that many false positives are due to natural ambiguities in the text and task description that are difficult to remove. A complementary finding that answers RQ2, which asks about the performance of dependency parsing alone, suggests that context and tacit knowledge are required to identify relevant actions and information type pairs. The crowd-worker annotations, which are reasonably low cost to acquire, can be used as guidance for selecting parser dependencies to identify a set of high confidence pairs. Our results also show that these high-confidence pairs contain most of the true positives as compared to the expert annotations, a minimal number of false positives that the hybrid approach identifies but were not identified by the experts, and omit a minimal number of false negatives that were identified by the expert annotators but missed by the hybrid approach.

The lexicon produced mixed results with respect to RQ3, which asks about the lexicon's utility to find missing annotations. The lexicon increased recall, but at a high cost of precision, because the lexicon lacks contextual cues to distinguish when particular action and information type phrases are true positives. In response to RQ4 about lexicon reuse and saturation, we observed that the lexicon reaches a saturation limit of between $42 \%$ and $84 \%$ in the domain of privacy policies, which suggests that the lexicon will likely never become complete. Alternatively, the lexicon may be used to find annotations for common words and phrases that can be used to further reduce the number of tasks sent to crowd workers and thus the overall framework cost, or can be used to solicit a higher number of workers to complete the reduced number of tasks for the same cost, thus reducing the probability of false negatives. 
In response to RQ5, which asks whether crowd workers can reduce these false positives, we observed an improvement in precision as we sent the selected high-confidence pairs back to the crowd for acceptance or rejection. Improvements in the front end of the framework (steps 3, 4, and B1 or B2) could further reduce the number of pairs that need to be sent to the workers in step 5, further improving the overall performance.

Based on our earlier work in crowd-worker goal annotations [Breaux and Schaub 2014 ], we estimate our target cost for extracting privacy goals from policies to be $\$ 0.92$ per statement, which is the cost of two experts to annotate the modality, information actions, information types, sources, targets, and purposes. There is an additional $\$ 0.18$ to have two experts formalize a statement, which aligns with task recomposition. Our results in Section 4 yield a partial specification (excluding the purposes) that costs $\$ 1.00$ per sentence, which is still under the $\$ 1.10$ total cost per statement that we obtained with expert annotators, but which excludes the work to identify and link purposes to the goal specifications. One promising observation is that purposes are fairly sparse in the dataset and conform to a particular phrase structure that may make them more amenable to automatic detection using phrase structure grammar patterns or machine learning. In addition, a significant portion of the increased cost comes from the validation task (step 5 in Figure 1). As shown in Table XI, there are still a large number of false positives in the direct and indirect high-confidence pairs. To the extent that we can reduce these false positives, we can further reduce the cost of crowd-worker extraction.

In our study, we examined privacy policies, which are legal documents intended to be read by various stakeholders, including website users, customers visiting "brickand-mortar" physical stores, and regulators. Although we cannot say with certainty, we believe that this approach could perform well on other information-related policies and laws. In steps 3 and 4 of our framework in Figure 1, we rely on crowd workers to read and interpret a text segment and to identify the actions and information types in the text. With respect to complex laws such as the Privacy Rule of the Health Insurance Portability and Accountability Act (HIPAA), our approach will be limited, because such laws rely on readers to integrate text from across multiple sections to frame the context (e.g., definitions and cross-references to exceptions). Therefore, to prepare the inputs for crowd workers, a trained analyst would need to "decontextualize" the legal text by systematically integrating relevant content from related sections of the law. If successful, however, we found that the crowd workers were able to identify both the implicit (e.g., provide, access) and explicit (e.g., collect, use, personal information) types of actions and information types with high recall as compared to the expert annotations. This variation in language use may be more frequent in policies than in laws, in which case, crowd workers may have an easier time identifying relevant noun and verb phrases in laws. For example, the narrower and more consistent use of terminology found in laws may improve the results of step B1 in Figure 1, which consists of the automation achieved by reusing a lexicon of established terminology. The lexicon may reach better saturation across multiple laws than what we observed in policies (see Figure 6), if those laws all adopt similar terms in their regulatory codes.

In summary, we introduced and evaluated a method that combines crowdsourcing and NLP to extract goals from privacy policies. The results show that crowd workers provide human interpretations that are still beyond the state of the art in NLP and that, for problems with predictable characteristics based on lexical or syntactic features, the NLP provides a cost-effective means to scale the extraction to a larger number of documents. We observed, however, that neither approach can be used by itself: the crowd workers vary in their interpretations and they are prone to miss or overlook information, whereas the NLP techniques studied cannot differentiate among the semantic cues needed to identify relevant phrases without the crowd workers. In 
the end, we observed that both classes of techniques are complementary and can be used to address each other's weaknesses for improved performance. While we believe this technique could be applied to other domains with similar results, future work is needed to evaluate our approach in such domains.

\section{ACKNOWLEDGMENTS}

We thank Hanan Hibshi, Sepideh Ghanavati, Daniel Smullen, Sudarshan Wadkar, and the CMU Requirements Engineering Lab for their helpful feedback.

\section{REFERENCES}

Russell J. Abbott. 1983. Program design by informal English descriptions. Communications of the ACM 26, 882-894. DOI:http://dx.doi.org/10.1145/182.358441

Annie I. Antón. 1997. Goal Identification and Refinement in the Specification of Information Systems. Ph.D. Thesis. Georgia Institute of Technology, Atlanta, GA.

Annie I. Antón and Julia B. Earp. 2004. A requirements taxonomy for reducing website privacy vulnerabilities. Requirements Engineering Journal 9, 3, 169-185. DOI:http://dx.doi.org/10.1007/s00766003-0183-z

Mohammad Allahbakhsh, Boualem Benatallah, Aleksandar Ignjatovic, Hamid Reza Motahari-Nezhad, Elisa Bertino, and Schahram Dustdar. 2013. Quality control in crowdsourcing systems: Issues and directions. IEEE Internet Computing 17, 2, 76-81. DOI:http://dx.doi.org/10.1109/MIC.2013.20

Paul André, Aniket Kittur, and Steven P. Dow. 2014. Crowd synthesis: Extracting categories and clusters from complex data. In Proceedings of the 17th ACM Conference on Computer Supported Cooperative Work and Social Computing. ACM, New York, NY, 989-998. DOI:http://dx.doi.org/10.1145/2531602.2531653

Daniel M. Berry, Erik Kamsties, and Michael M. Krieger. 2003. From Contract Drafting to Software Specification: Linguistic Sources of Ambiguity. Technical Report. University of Waterloo, Waterloo, ON.

Daniel M. Berry, Ricardo Gacitua, Pete Sawyer, and Sri Fatimah Tjong. 2012. The case for dumb requirements engineering tools. In Proceedings of the 18th International Conference on Requirements Engineering: Foundation for Software Quality. Björn Regnell and Daniela Damian (Eds.). Springer, Berlin, 211-217. DOI:10.1007/978-3-642-28714-5_18.

Jaspreet Bhatia and Travis D. Breaux. 2015. Towards an information type lexicon for privacy policies. 8th IEEE International Workshop on Requirements Engineering and Law. IEEE Computer Society, Washington, DC, 19-24. DOI:10.1109/RELAW.2015.7330207.

Travis D. Breaux and Ashwini Rao. 2013. Formal analysis of privacy requirements specifications for multitier applications. In 21st IEEE International Requirements Engineering Conference (RE'13). Rio de Janeiro, Brazil, 14-23.

Travis D. Breaux and Florian Schaub. 2014. Scaling requirements extraction to the crowd: Experiments on privacy policies. $22^{\text {nd }}$ IEEE International Requirements Engineering Conference. IEEE Computer Society, Washington, DC, 163-172. DOI:10.1109/RE.2014.6912258

Travis D. Breaux, Annie I. Antón, and Jon Doyle. 2008. Semantic parameterization: A process for modeling domain descriptions. ACM Transactions on Software Engineering Methodology 18, 2, Article 5, 27. DOI:http://dx.doi.org/10.1145/1416563.1416565

Travis D. Breaux, Hanan Hibshi, and Ashwini Rao. 2014. Eddy, a formal language for specifying and analyzing data flow specifications for conflicting privacy requirements. Requirements Engineering Journal 19, 3, 281-307. DOI:http://dx.doi.org/10.1007/s00766-013-0190-7

Travis D. Breaux, Daniel Smullen, and Hanan Hibshi. 2015. Detecting repurposing and over-collection in multi-party privacy requirements specifications. $23^{\text {rd }}$ IEEE International Requirements Engineering Conference. IEEE Computer Society, Washington, DC, 166-175. DOI:10.1109/RE.2015.7320419

Erik Casagrande, Selamawit Woldeamlak, Wei Lee Woon, H. H. Zeineldin, and Davor Svetinovic. 2014. NLPKAOS for systems goal elicitation: Smart metering system case study. IEEE Transactions in Software Engineering 40, 10, 941-956. DOI:10.1109/TSE.2014.2339811

Michelene T. H. Chi, Paul J. Feltovich, and Robert Glaser. 1981. Categorization and representation of physics problems by experts and novices. Cognitive Science 5, 2, 121-152. DOI:10.1207/s15516709cog0502_2

Anne Dardenne, Axel van Lamsweerde, and Stephen Fickas. 1993. Goal-directed requirements acquisition. In Selected Papers of the 6th International Workshop on Software Specification and Design, M. Sintzoff, C. Ghezzi, and G. C. Roman (Eds.). Elsevier Science Publishers B. V., Amsterdam, The Netherlands, 3-50. DOI:10.1016/0167-6423(93)90021-G 
Marie-Catherine de Marneffe, Bill MacCartney, and Christopher D. Manning. 2006. Generating typed dependency parses from phrase structure parses. In Proceedings of International Conference on Language Resources and Evaluation, 449-454.

Nguyen Q. V. Hung, Nguyen T. Tam, Lam N. Tran, and Karl Aberer. 2013. An evaluation of aggregation techniques in crowdsourcing. Web Information Systems Engineering. Springer, Berlin, 1-15. DOI:10.1007/9783-642-41154-0_1

Silvia Ingolfo, Ivan Jureta, Alberto Siena, Anna Perini, and Angelo Susi. 2014. Nòmos 3: Legal compliance of roles and requirements. Conceptual Modeling. Lecture Notes in Computer Science, Vol. 8824, $275-288$. DOI:10.1007/978-3-319-12206-9_22

John S. Justeson and Slava M. Katz. 1995. Technical terminology: Some linguistic properties and an algorithm for identification in text. In Natural Language Engineering 1, 1, 9-27. D0I:10.1017/ S1351324900000048

Erik Kamsties. 2006. Understanding ambiguity in requirements engineering. Engineering and Managing Software Requirements. Springer, The Netherlands, 245-266. DOI:10.1007/3-540-28244-0_11

Aniket Kittur, Boris Smus, Susheel Khamkar, and Robert E. Kraut. 2011. CrowdForge: Crowdsourcing complex work. In Proceedings of the 24th Annual ACM Symposium on User Interface Software and Technology. ACM, New York, NY, 43-52. DOI:http://dx.doi.org/10.1145/2047196.2047202

Leonid Kof. 2004. Natural language processing for requirements engineering: Applicability to large requirements documents. In Proceedings of the 19th International Conference on Automated Software Engineering Workshops, 91-102.

Soo L. Lim and Anthony Finkelstein. 2012. StakeRare: Using social networks and collaborative filtering for large-scale requirements elicitation. IEEE Transactions on Software Engineering 38, 3, 707-735. DOI:http://dx.doi.org/10.1109/TSE.2011.36

Jialiu Lin, Shahriyar Amini, Jason I. Hong, Norman Sadeh, Janne Lindqvist, and Joy Zhang. 2012. Expectation and purpose: Understanding users' mental models of mobile app privacy through crowdsourcing. In Proceedings of the 2012 ACM Conference on Ubiquitous Computing. ACM, New York, NY, 501-510. DOI:http://dx.doi.org/10.1145/2370216.2370290

Lin Liu, Tianying Li, and Xiaoxi Kou. 2014. Eliciting relations from natural language requirements documents based on linguistic and statistical analysis. $38^{\text {th }}$ IEEE Annual Computer Software and Applications Conference, 191-200. DOI:10.1109/COMPSAC.2014.27

Aleecia M. McDonald and Lorrie F. Cranor. 2008. The cost of reading privacy policies. I/S: A Journal of Law and Policy for the Information Society 4, 3, 540-565.

Paul N. Otto, Annie I. Anton, and David L. Baumer. 2007. The choicepoint dilemma: How data brokers should handle the privacy of personal information. IEEE Security and Privacy 5, 5, $15-23$. DOI:10.1109/MSP.2007.126

Daniel Popescu, Spencer Rugaber, Nenad Medvidovic, and Daniel M. Berry. 2008. Reducing ambiguities in requirements specifications via automatically created object-oriented models. In Innovations for Requirement Analysis. From Stakeholders' Needs to Formal Designs, Barbara Paech and Craig Martell (Eds.). Lecture Notes in Computer Science, Vol. 5320. Springer, Berlin, 103-124. DOI:http://dx.doi.org/10.1007/978-3-540-89778-1_10

Elda Paja, Fabiano Dalpiaz, and Paolo Giorgini. 2015. Modeling and reasoning about security requirements in socio-technical systems. Data Knowledge Engineering 98, C, 123-143. D0I:http://dx.doi.org/ 10.1016/j.datak.2015.07.007

Alexander J. Quinn and Benjamin B. Bederson. 2011. Human computation: A survey and taxonomy of a growing field. In Proceedings of the SIGCHI Conference on Human Factors in Computing Systems. ACM, New York, NY, 1403-1412. DOI:http://dx.doi.org/10.1145/1978942.1979148

J. R. Reidenberg, T. D. Breaux, L. F. Cranor, B. French, A. Grannis, J. T. Graves, F. Liu, A. M. McDonald, T. B. Norton, R. Ramanath, N. C. Russell, N. Sadeh, and F. Schaub. 2015. Disagreeable privacy policies: Mismatches between meaning and users' understanding. Berkeley Technology Law Journal 30, 1, 39-88.

Eleanor Rosch, Carolyn B. Mervisa, Wayne D Gray, David M Johnson, and Penny Boyes-Braem. 1976. Basic objects in natural categories. Cognitive Psychology 8, 3, 382-439. DOI:10.1016/0010-0285(76)90013-X

Marta Sabou, Kalina Bontcheva, and Arno Scharl. 2012. Crowdsourcing research opportunities: Lessons from natural language processing. In Proceedings of the 12th International Conference on Knowledge Management and Knowledge Technologies. ACM, New York, NY, Article 17, 8. DOI:http://dx.doi.org/ $10.1145 / 2362456.2362479$

Patrick Shafto and John D. Coley. 2003. Development of categorization and reasoning in the natural world: Novices to experts, naive similarity to ecological knowledge. Journal of Experimental Psychology: Learning Memory and Cognition 29, 4, 641-649. DOI:10.1037/0278-7393.29.4.641 
Rion Snow, Brendan O’Connor, Daniel Jurafsky, and Andrew Y. Ng. 2008. Cheap and fast-but is it good?: Evaluating non-expert annotations for natural language tasks. In Proceedings of the Conference on Empirical Methods in Natural Language Processing. Association for Computational Linguistics, Stroudsburg, PA, 254-263.

James W. Tanaka and Marjorie Taylor. 1991. Object categories and expertise: Is the basic level in the eye of the beholder? Cognitive Psychology 23, 3, 457-482.

Vasilis Verroios and Michael S. Bernstein. 2014. Context trees: Crowdsourcing global understanding from local views. In 2nd AAAI Conference on Human Computation and Crowdsourcing, 210-219.

Wesley Willett, Jeffrey Heer, and Maneesh Agrawala. 2012. Strategies for crowdsourcing social data analysis. In Proceedings of the SIGCHI Conference on Human Factors in Computing Systems. ACM, New York, NY, 227-236. DOI:http://dx.doi.org/10.1145/2207676.2207709

Hui Yang, Alistair Willis, Anne De Roeck, and Bashar Nuseibeh. 2010. Automatic detection of nocuous coordination ambiguities in natural language requirements. In Proceedings of the IEEE/ACM International Conference on Automated Software Engineering. ACM, New York, NY, 53-62. DOI:http://dx.doi.org/ 10.1145/1858996.1859007

Hui Yang, Anne de Roeck, Vincenzo Gervasi, Alistair Willis, and Bashar Nuseibeh. 2011. Analysing anaphoric ambiguity in natural language requirements. Requirements Engineering Journal 16, 3, 163189. DOI:http://dx.doi.org/10.1007/s00766-011-0119-y

Robert. K. Yin. 2009. Case study research (4th ed.). In Applied Social Research Methods Series, Vol. 5. Sage Publications.

Jing Zhang, Xindong Wu, and Victor S. Sheng. 2013. A threshold method for imbalanced multiple noisy labeling. In Proceedings of the 2013 IEEE/ACM International Conference on Advances in Social Networks Analysis and Mining. ACM, New York, NY, 61-65. DOI:http://dx.doi.org/10.1145/2492517.2492640

Received October 2015; revised February 2016; accepted March 2016 